\title{
Cell vacuolation induced by Haemophilus influenzae supernatants in HEp-2 cells
}

\author{
María del Rosario Espinoza-Mellado ${ }^{1 /+}$, Edgar Oliver López-Villegas², \\ Ramón I Arteaga-Garibay³, Silvia Giono-Cerezo'

\begin{abstract}
'Laboratorio de Bacteriología Médica, Departamento de Microbiología ${ }^{2}$ Central de Instrumentación de Microscopía, Departamento de Investigación, Escuela Nacional de Ciencias Biológicas, Instituto Politécnico Nacional, México DF, México ${ }^{3}$ Laboratorio de Recursos Genéticos Microbianos, Centro Nacional de Recursos Genéticos, Instituto Nacional de Investigaciones Forestales y Agropecuarias, Tepatitlán, Jalisco, México
\end{abstract}

Haemophilus influenzae belongs to respiratory tract microbiota. We observed vacuoles formation in previous studies with $\mathrm{H}$. influenzae culture supernatants, so in this work we characterised that cytotoxic effect. We observed an abundant production of acidic cytoplasmic vacuoles due to the presence of a "vacuolating factor" in $\mathrm{H}$. influenzae supernatants which was characterised as thermolabile. Greatest vacuolating activity was observed when utilizing the fraction $>50 \mathrm{kDa}$. The presence of a large number of vacuoles in HEp-2 cells was verified by transmission electron microscopy and some vacuoles were identified with a double membrane and/or being surrounded by ribosomes. These results suggest similar behaviour to that of vacuolating effects described by autotransporter proteins an undescribed cytotoxic effect induced by $\mathrm{H}$. influenzae.

Key words: Haemophilus influenzae - cell vacuolation - cytotoxicity assays

Haemophilus influenzae is a human pathogen; at present, none of its virulence factors has been described with vacuolating cytotoxic activity on host cells (Moxon 2009, Kostyanev \& Sechanova 2012). Hap is an autotransporter protein of $H$. influenzae that shares features with vacuolating proteins, such as serine protease activity (Yen et al. 2008). Several authors had been worked on characterisations of bacterial supernatants and some proteins which possess vacuolating activity have been described, such as: Vat, Sat, Pet, PicU and EspC proteins of Escherichia coli (Guyer et al. 2002), vacuolating cytotoxic factor in Aeromonas veronii bt. sobria (Martins et al. 2007), ShlA of Serratia marcenscens, HlyA of Vibrio cholerae and VacA of Helicobacter pylori (FigueroaArredondo et al. 2001, Vidal et al. 2009). The aim of this study was to characterise the vacuolating effect induced by $H$. influenzae supernatants of ATCC strains, since this could be an undescribed pathogenic mechanism related with the vacuolating activity of certain autotransporter proteins, as a cell response against intracellular pathogens and also could be important for removing damaged organelles.

doi: 10.1590/0074-0276130716

Financial support: SIP-IPN (20100738, 20110312, 20120875,

20130644), ENCB-IPN

MRE-M has been the recipient of a scholarship from CONACyT-

México and CARSO Institute since 2009 and SG-C is the recipient of SNI, COFAA and EDI fellowships.

+ Corresponding author: chayocobain@gmail.com

Received 27 December 2012

Accepted 2 October 2013
H. influenzae ATCC 10211, 49766, 49247 and 33930 strains were used. E. coli $933 \mathrm{~W}$ and $E$. coli $\mathrm{K} 12$ strains were used as cytotoxicity controls. Brain heart infusion broth supplemented was employed as the culture medium and incubation was done for $24 \mathrm{~h}$ at $37^{\circ} \mathrm{C}$. The ATCC CCL23 HEp-2 cell line (human larynx carcinoma) was grown as previously described by St Geme et al. (1993). We defined culture supernatants as the result of centrifuge the $H$. influenzae culture and separate it of the bacterial pellet and were obtained at incubation time periods, including $12 \mathrm{~h}, 15 \mathrm{~h}, 18 \mathrm{~h}, 21 \mathrm{~h}$ and 24 $\mathrm{h}$, were filtered with $0.22-\mu \mathrm{m}$ Millipore ${ }^{\circledR}$ nitrocellulose membranes. Protein concentration was measured by Bradford method and all the supernatants were adjusted to a concentration of $0.15-0.2 \mathrm{mg} / \mathrm{mL}$.

Cytotoxicity assays were performed according to Guyer et al. (2002) and Arellano-Galindo et al. (2007), where $H$. influenzae supernatants were inoculated into a HEp-2 cell monolayer in a 24 -well microplate and incubated $2 \mathrm{~h}$ at $37^{\circ} \mathrm{C}$. Cell vacuolation was observed at $1 \mathrm{~h}, 2$ $\mathrm{h}, 3.5 \mathrm{~h}$ and $24 \mathrm{~h}$. Fifty percent cytotoxic dose (CD50\%) was determined by the Probit analysis using SPSS 17.0 software and titre was defined as the reciprocal of the highest dilution that shows $50 \%$ vacuolation of HEp-2 cells. Cells were washed, fixed, stained with Giemsa dye and observed by optical microscope. Vacuolation induced was analysed by transmission electron microscopy (TEM). Cytotoxic effect was defined as any morphological change and vacuolating effect was defined as a cytoplasmic vacuoles formation, both after treatment.

The characterisation of "vacuolating factor" in supernatants included thermal stability assays in which supernatants were treated at several temperatures by $15 \mathrm{~min}$, as described by Arellano-Galindo et al. (2007). Neutral red dye uptake of vacuoles was determined as described 
Figueroa-Arredondo et al. (2001). For the protease assay, H. influenzae ATCC 33930 supernatant was treated with $2 \mathrm{U}$ of Proteinase $\mathrm{K}$ Roche ${ }^{\circledR}$ at $37^{\circ} \mathrm{C}$ as described by Mitra et al. (2000); that culture supernatant was concentrated also, in order to estimate molecular size, utilizing the Amicon $^{\circledR}$ Ultra centrifugal filter $50 \mathrm{kDa}$ cut-off (Millipore ${ }^{\circledR}$ ) according to Vidal and Navarro-García (2006). The vacuolating activity after each treatment was performed under the aforementioned conditions described. Mann Whitney $U$ test $(p<0.05)$ was used to assess the differences in vacuolation between strains.

We found cytotoxic effects induced in HEp-2 cells using $H$. influenzae supernatants, such as cell vacuolation (Supplementary data), cytoplasm deformation, nucleus condensation and monolayer destruction. Vacuolation was observed at the highest percentage of cells (52-89\%) compared with the others. Incubation time to obtain active culture supernatants was $21 \mathrm{~h}$ and it is agree with Wang et al. (1996), in which cytotoxin production depends on bacteria growth. Statistical analyses (Fig. 1) corroborated that cell vacuolation percentages showed significant differences comparing with negative control $(\mathrm{p}<0.05)$. We observed HEp-2 cells cytolysis after continuous exposure to supernatants. It was proposed that cell vacuolation is a stage prior to cytolysis (FigueroaArredondo et al. 2001), which was evidenced when cells were detached from the monolayer. Therefore, this showed that continuous exposure to the $H$. influenzae "vacuolating factor" can result in cell death. Vacuolation comprises extensive cell injury that has been described for many bacteria (Vidal et al. 2009). Therefore, host-cell vacuolation could be considered as a conserved pathogenic mechanism among certain medically important bacteria, which could improve their pathogenicity and establishment in host cells. H. influenzae strains ATCC 49247 and ATCC 49766 had the highest CD50\% value, $1: 158.75$ and 1:125.27 $(\mathrm{p}<0.1)$, respectively; thus, they exhibited high vacuolating activity. These results demonstrated the variety of titres that could be obtained in the evaluation of the biological vacuolating activity of different bacterial supernatants. These data are in agreement with previous reports, which showed that some pathogens produce toxins that affect host-cell stability and that could be present in bacterial supernatants (Vidal et al. 2009) so we propose the presence of a "vacuolating factor" in $H$. influenzae supernatants.

TEM analysis was used to ratify the presence of cytoplasmic vacuoles elicited by $H$. influenzae supernatants. We observed no changes in cellular organelles, only some disruptions in the cytoplasmic cell membrane, a few plasma membrane projections and loose chromatin. TEM images showed that cells contained a large number of vacuoles and, in certain sections, the vacuoles appeared to be continuous with the cell membrane system or to show a double membrane. We did not find difference between morphology of vacuoles induced by all the supernatants (Fig. 2). Induced vacuoles occupied a considerable area within HEp-2 cytoplasm and, in comparison with vacuoles observed in autophagy (Kroemer \& Levine 2008), appear to share some characteristics,

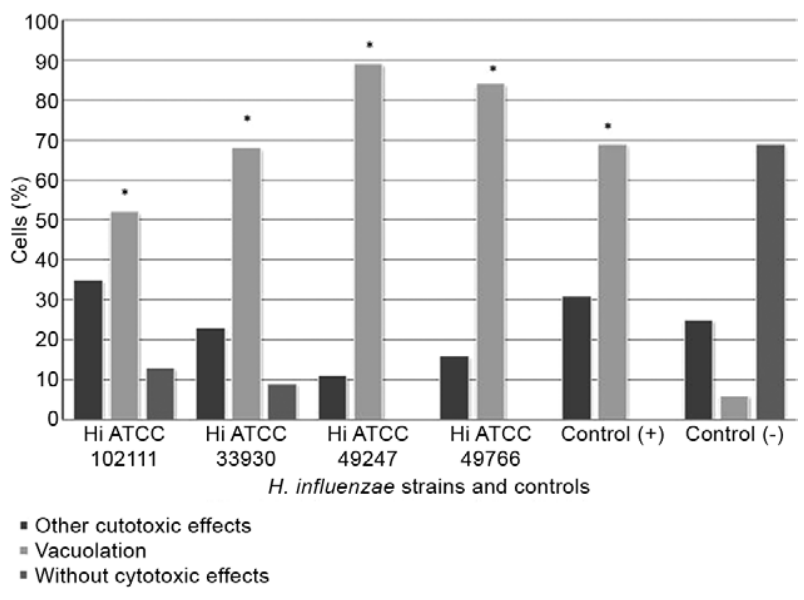

Fig. 1: cytotoxic vacuolating effect induced by Haemophilus influenzae (Hi) active supernatants on HEp-2 cells. "Other cytotoxic effects" means other cytotoxic effects different to vacuolation effect, as nuclear fragmentation, nucleus condensation and cytoplasm deformation. Asterisk means that the value has a statistically significant difference compared with the negative control $(\mathrm{p}<0.05)$. Cytotoxicity percentages were determined by counting 100 cells in different fields of the sample.

such as double-membrane vacuoles. Ribosomes were identified in the vacuole periphery, suggesting a possible origin of rough endoplasmic reticulum; the intense cytoplasm vacuolation observed could be similar to the effect caused by aforementioned autotransporter proteins and autophagy has been observed occurring in cells intoxicated by some of them (Moal et al. 2011). According to our viewpoint, the images obtained suggest an autophagosome formation; while, TEM is a good method for screening (Eskelinen et al. 2011), is important to support these results with more specific techniques to elucidate whether the autophagic process is related with the cytotoxic damage caused by $H$. influenzae supernatants, because it has not yet been described as a mechanism of injury or as a response to $H$. influenzae infections, in which autophagy could play a protective role for hostcells attempting self-limit damage as well as promoting bacterium persistence (Wirawan et al. 2012).

The "vacuolating factor" was thermolabile, since vacuolating effect was lost at $65^{\circ} \mathrm{C}$; this behaviour could be similar to that of some vacuolating cytotoxins, as cited Arellano-Galindo et al. (2007) and Martins et al. (2007). The majority of vacuoles induced by the $H$. influenzae "vacuolating factor" captured neutral red (acidic microenvironment) (Supplementary data), suggesting that they probably derived from late or pre-lysosomal endosomes, similar to the VacA protein (Figueroa-Arredondo et al. 2001). We did not observe residual activity of supernatants treated with proteinase, so we could suggest that "vacuolating factor" was proteinaceous. Similar results have been demonstrated with $V$. cholerae supernatants in order to recognise haemolysin as the responsible of vacuolating effects observed on that model (Mitra et al. 2000). Fractions of $>50 \mathrm{kDa}$ of supernatant produced 

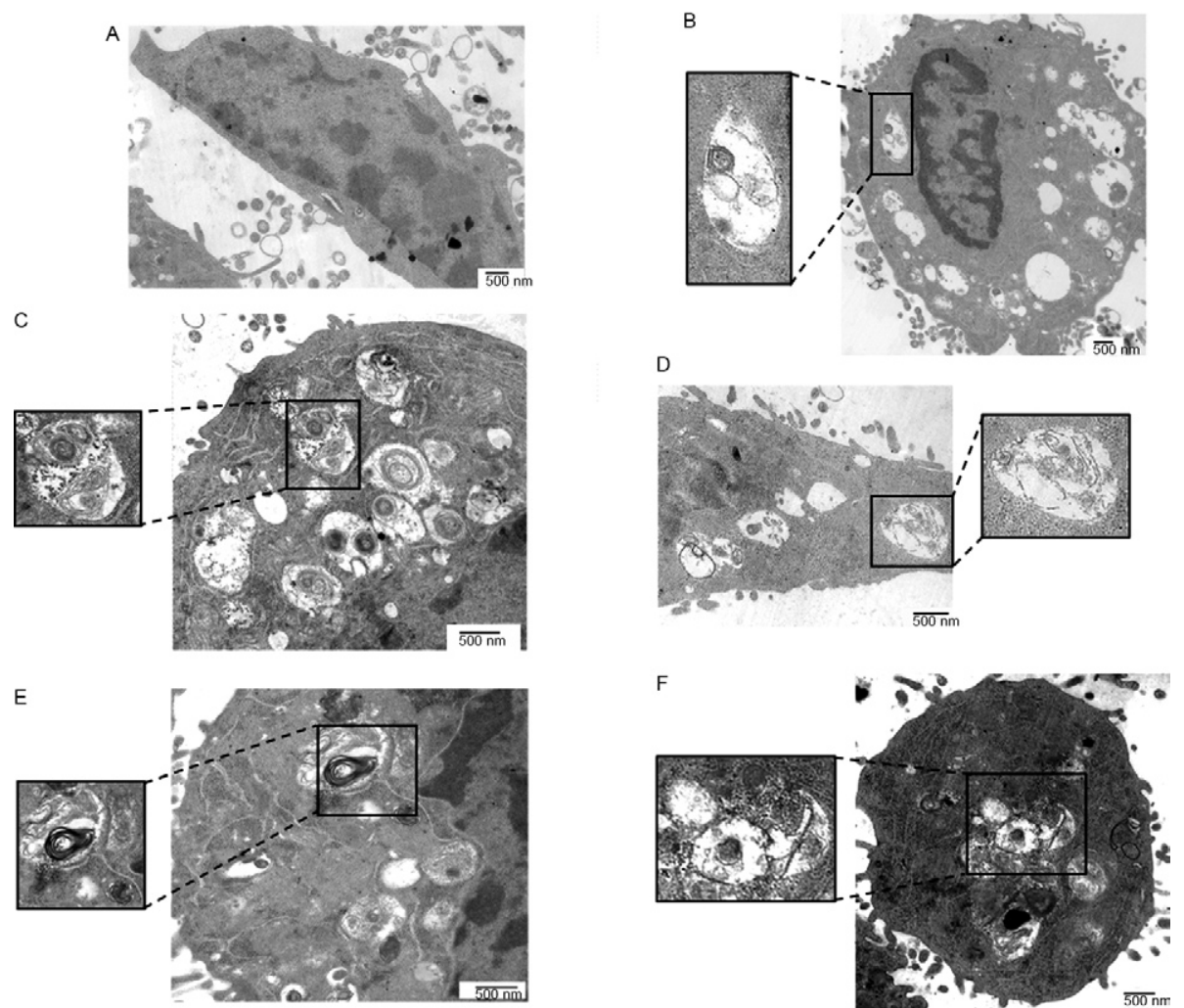

Fig. 2: transmission electron microscopy images presenting cytotoxic damage in HEp-2 cells induced by Haemophilus influenzae supernatants. A: Escherichia coli K12 negative control, magnification 25,000X; B: H. influenzae ATCC 49247, magnification 15,000X, 20,000X; C: E. coli 933W positive control, magnification 20,000X, 40,000X; D: H. influenzae ATCC 10211, magnification 25,000X, 40,000X; E: H. influenzae ATCC 49766, magnification 25,000X, 40,000X; F: H. influenzae ATCC 33930, magnification 20,000X, 25,000X.

intense cell vacuolation, with percentages ranging from $68-80 \%$. Several autotransporter proteins possessing vacuolating activity were described in $E$. coli, in which their sizes were around $100 \mathrm{kDa}$ and $\mathrm{Hap}_{\mathrm{s}}$ protein has a size of $110 \mathrm{kDa}$ (Yen et al. 2008); therefore, research on $H$. influenzae $\mathrm{Hap}_{\mathrm{S}}$ residual activity would be important for understanding whether this virulence factor could be responsible for the cytotoxic effect observed. Structural similarity between VacA, $H$. influenzae IgA protease and Hap protein has been reported (Schmitt \& Haas 1994, Kilian et al. 2002); therefore, it may be possible that Hap could share some functions with the VacA protein. Further experiments must be realised to elucidate if there is a relation between autotransporter protein family and vacuolating activity.

This paper could open a new way to understand which "vacuolating factor" is produced by $H$. influenzae. Several authors have commented the relevance of identify at first, cytotoxic and vacuolating activity in culture supernatants of bacterial strains, in order to characterise it after (Vidal et al. 2009).

\section{ACKNOWLEDGEMENTS}

To CV Jardón-Serrano, for critical reading of the manuscript, to V Flores-López, for the help with the statistical data analysis.

\section{REFERENCES}

Arellano-Galindo J, Rodríguez-Angeles MG, Velázquez-Guadarrama N, Santos-Esteban E, Giono-Cerezo S 2007. Evaluation of in vivo and in vitro biological activity of a Vibrio cholerae 01 hemolysin. Clin Invest Med 30: 101-107.

Eskelinen EL, Reggiori F, Baba M, Kovács AL, Seglen PO 2011. Seeing is believing. The impact of electron microscopy on autophagy research. Autophagy 7: 935-956.

Figueroa-Arredondo P, Heuser JE, Akopyants NS, Morisaki JH, Giono-Cerezo S, Enríquez-Rincón F, Berg DE 2001. Cell vacuolation caused by Vibrio cholerae hemolysin. Infect Immun 69: 1613-1624.

Guyer DM, Radulovic S, Jones FE, Mobley HLT 2002. Sat, the secreted autotransporter toxin of uropathogenic Escherichia coli is a vacuolating cytotoxin for bladder and kidney epithelial cells. Infect Immun 70: 4539-4546.

Kilian M, Poulsen K, Lomholt H 2002. Evolution of the paralogous hap and iga genes in Haemophilus influenzae: evidence for a conserved hap pseudogene associated with microcolony formation in the recently diverged Haemophilus aegyptius and $H$. influenzae biogroup aegyptius. Mol Microbiol 46: 1367-1380.

Kostyanev TS, Sechanova LP 2012. Virulence factors and mechanisms of antibiotic resistance of Haemophilus influenzae. Folia Med (Plovdiv) 54: 19-23.

Kroemer G, Levine B 2008. Autophagic cell death: the story of a misnomer. Nat Rev Mol Cell Biol 9: 1004-1010. 
Martins LM, Catani CF, Falcón RM, Carbonell GV, Azzoni AA, Yano $\mathrm{T}$ 2007. Induction of apoptosis in Vero cells by Aeromonas veronii biovar sobria vacuolating cytotoxic factor. FEMS Immunol Med Microbiol 49: 197-204.

Mitra R, Figueroa P, Mukhopadhyay AK, Shimada T, Takeda Y, Berg DE, Nair GB 2000. Cell vacuolation, a manifestation of the ElTor hemolysin of Vibrio cholerae. Infect Immun 68: 1928-1933.

Moal VL, Comenge Y, Ruby V, Amsellem R, Nicolas V, Servin AL 2011. Secreted autotransporter toxin (Sat) triggers autophagy in epithelial cells that relies on cell detachment. Cell Microbiol 13: 992-1013.

Moxon ER 2009. Bacterial variation, virulence and vaccines. Microbiology 155: 997-1003.

Schmitt W, Haas R 1994. Genetic analysis of the Helicobacter pylori vacuolating cytotoxin: structural similarities with the $\operatorname{IgA}$ protease type of exported protein. Mol Microbiol 12: 307-319.

St Geme JW 3rd, Falkow S, Barenkamp SJ 1993. High-molecularweight proteins of nontypable Haemophilus influenzae mediate attachment to human epithelial cells. Proc Natl Acad Sci USA 90: 2875-2879.

Vidal JE, Enríquez-Rincón F, Giono-Cerezo S, Ribas-Aparicio RM, Figueroa-Arredondo P 2009. Culture supernatants from $V$. cholerae $\mathrm{O} 1 \mathrm{ElTor}$ strains isolated from different geographic areas induce cell vacuolation and cytotoxicity. Salud Publica Mex 51: 39-47.

Vidal JE, Navarro-García F 2006. Efficient translocation of EspC into epithelial cells depends on enteropathogenic Escherichia coli and host cell contact. Infect Immun 74: 2293-2303.

Wang G, Tyler KD, Munro CK, Johnson WM 1996. Characterization of cytotoxic, hemolytic Aeromonas caviae clinical isolates and their identification by determining presence of a unique hemolysin gene. J Clin Microbiol 34: 3203-3205.

Wirawan E, Berghe TV, Lippens S, Agostinis P, Vandenabeele P 2012. Autophagy: for better or for worse. Cell Res 22: 43-61.

Yen YT, Kostakioti M, Henderson IR, Stathopoulos C 2008. Common themes and variations in serine protease autotransporters. Trends Microbiol 16: 370-379. 


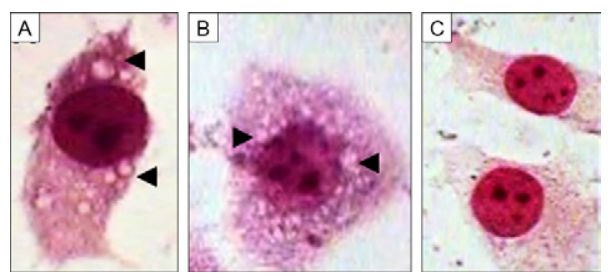

Cytotoxic effects on HEp-2 cells induced by contact with Haemophilus influenzae supernatants obtained after $21 \mathrm{~h}$ of incubation. A: H. influenzae ATCC 10211; B: H. influenzae ATCC 49247; C: HEp-2 cell control. Arrowheads indicate cytoplasmic vacuoles. Magnification 100X.

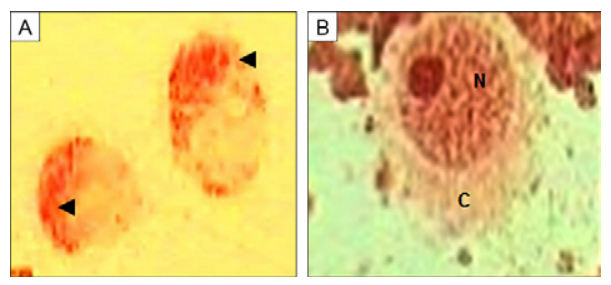

Neutral red uptake in vacuoles induced by Haemophilus influenzae supernatants on HEp-2 cells. A: H. influenzae ATCC 33930; B: Escherichia coli K12 negative control; C: cytoplasm; N: nucleus. Arrowheads indicate acidic vacuoles. Magnification 100X. 